\title{
RELAÇÕES ENTRE MATÉRIA ORGÂNICA DO SOLO E DECLIVIDADE DE VERTENTES EM TOPOSSEQÜÊNCIA DE LATOSSOLOS DO SUL DE MINAS GERAIS ${ }^{(1)}$
}

\author{
Alexandre Christofaro Silva ${ }^{(2)}$, Pablo Vidal Torrado ${ }^{(3)}$, \\ Martha González Pérez ${ }^{(4)}$, Ladislau Martin Neto ${ }^{(4)}$ \& \\ Felipe Macias Vasquez ${ }^{(5)}$
}

\begin{abstract}
RESUMO
O acúmulo e a estabilidade da matéria orgânica do solo estão relacionados com a declividade de vertentes. Por essa razão, foram avaliadas quantitativamente relações entre a declividade e o grau de humificação de ácidos húmicos, o conteúdo de fragmentos de carvão e suas idades radiocarbônicas. Foram amostrados quatro Latossolos situados nas posições de topo, ombro, meia encosta e sopé de uma toposseqüência com pastagens, de uma área cratônica do Sul de Minas Gerais. Os Latossolos são originados de gnaisses do Complexo Cristalino, da Era PréCambriana, e apresentam regime hídrico údico e regime térmico isotérmico. Foram coletadas amostras de horizontes e sub-horizontes dos quatro perfis, para caracterização química e física, extração de ácidos húmicos e determinação do conteúdo dos radicais livres do tipo semiquinona. Fragmentos de carvão foram coletados nesses mesmos sub-horizontes, para quantificação gravimétrica, avaliação dos teores de $\mathrm{C}$ e $\mathrm{N}$ e para datações radiocarbônicas. Os resultados deste estudo demonstram que, em toposseqüências de Latossolos do Sul de Minas Gerais, a acumulação e o grau de humificação da matéria orgânica do solo estão fortemente
\end{abstract}

\footnotetext{
(1) Trabalho apresentado no V International Meeting of International Humics Substances Society, em 2004. Parcialmente financiado pela FAPEMIG. Recebido para publicação em outubro de 2005 e aprovado em maio de 2007.

${ }^{(2)}$ Professor da Universidade Federal dos Vales do Jequitinhonha e Mucuri - UFVJM, Caixa Postal 38, CEP 39100-000 Diamantina (MG). E-mail: christo@jknet.com.br

(3) Professor do Departamento de Solos e Nutrição de Plantas, Escola Superior de Agricultura Luiz de Queiroz - ESALQ/USP. Caixa Postal 09, CEP 13418-900 Piracicaba (SP). E-mail: pablo@esalq.usp.br

(4) Pesquisador da Embrapa Instrumentação Agropecuária. Caixa Postal 741, CEP 13560-970 São Carlos (SP). E-mail: martin@cnpdia.embrapa.br

${ }^{(5)}$ Professor do Departamento de Edafoloxia e Facultad de Bioloxia, Universidad de Santiago de Compostela. CP 15706, Santiago de Compostela, Espanha. E-mail: edfmac@usc.es
} 
correlacionados com a declividade da vertente, enquanto a quantidade e a idade radiocarbônica de fragmentos de carvão estão relacionadas a processos de gênese dos solos e à posição na vertente.

Termos de indexação: ácidos húmicos do solo, carvão do solo, grau de humificação, EPR, datação radiocarbônica.

\title{
SUMMARY: RELATIONSHIP BETWEEN SOIL ORGANIC MATTER AND SLOPE STEEPNESS IN A TOPOSEQUENCE OF OXISOL IN SOUTHERN MINAS GERAIS, BRAZIL
}

\begin{abstract}
The accumulation and stability of soil organic matter are related to slope steepness. The relationships between steepness and the humification degree of humic acids, the charcoal fragment content and its radiocarbon age were quantitatively evaluated. Four Latosols (Oxisols) of the summit, shoulder, back slope and back/foot slope of a toposequence under pasture in a cratonic area in the southern Minas Gerais state, southwest Brazil, were used. The parent material of the Latosols was gneiss of the Precambrian complex, with an udic hydric regime and isothermic thermic regime. Samples were collected from every horizon and subhorizon of the four Latosols for chemical and physical characterization, humic acid extraction and determination of the semiquinone free radical content. Likewise, charcoal fragments were collected for gravimetric, nitrogen and carbon content quantification and radiocarbon dating. Our results show the close relationship of the accumulation and humification degree of soil organic matter in Latosol landscapes to slope steepness, whereas the quantity and radiocarbon age of charcoal fragments were related to the processes of soil genesis and soil position in the slope.
\end{abstract}

Index terms: soil humic acids, soil charcoal, humification degree, EPR, ${ }^{14} \mathrm{C}$ dating.

\section{INTRODUÇÃO}

O relevo é o fator de formação que controla a distribuição de matéria no sistema solo. A utilização de seus atributos, como a declividade, para estabelecer relações com os processos pedogenéticos tem facilitado o entendimento da pedogênese. O acúmulo e o grau de humificação da matéria orgânica, por exemplo, correlacionam-se com a declividade do terreno (Buol et al., 1980; Jenny, 1980; Curi \& Franzmeier, 1984; Lepsch \& Buol, 1986; Daniels \& Hammer, 1992; Silva \& Vidal Torrado, 1999; Silva et al., 2002). Geralmente, em áreas de boa drenagem e mantidos constantes os demais fatores de formação dos solos, quanto menor a declividade, maior é a taxa de acúmulo e o grau de humificação da matéria orgânica.

No topo plano de vertentes ocupadas por solos acentuadamente drenados, como os Latossolos, a erosão hídrica é incipiente, o que favorece o acúmulo de matéria orgânica, que, com o passar do tempo, vai sendo cada vez mais humificada. Na meia encosta dessas vertentes, a erosão hídrica remove parcialmente a matéria orgânica do solo, dificultando seu acúmulo e sua humificação (Buol et al., 1980; Bertoni \& Lombardi Neto, 1995).

Dessa forma, o conteúdo de C orgânico seria maior e a estrutura da matéria orgânica mais complexa no topo plano de uma vertente formada por Latossolos, ao passo que na meia encosta o conteúdo de $\mathrm{C}$ seria menor e a estrutura da matéria orgânica seria mais simples.

Silva (1997) e Silva \& Vidal Torrado (1999), estudando vertentes atualmente estáveis pertencentes a uma área cratônica do Sudeste do Brasil, reportaram teores de $\mathrm{C}$ orgânico mais elevados em perfis de solo situados no topo da vertente, enquanto nos perfis de solo situados na meia encosta esses teores foram menores. Nesses perfis, fragmentos de carvão são comumente encontrados em diferentes profundidades.

Fragmentos de carvão são de ocorrência comum em muitos solos tropicais, particularmente em Latossolos (Sanford et al., 1985; Bassini \& Becker, 1990; Boulet et al., 1995; Behling 1997a,b; Silva \& Vidal Torrado, 1999; Barberi et al., 2000; Gouveia, 2001). Seus conteúdos e suas idades radiocarbônicas mostraram-se mais altos em Latossolos situados em topos de menor declividade e mais baixos naqueles localizados em segmentos de vertente de maior declividade (Bassini \& Becker, 1990; Pessenda et al., 1996; Silva \& Vidal Torrado, 1999), já que estariam menos sujeitos à erosão hídrica e permaneceriam na paisagem por mais tempo.

Riffaldi \& Schnitzer (1972) reportam que radicais livres do tipo semiquinona são estabilizados como 
estruturas aromáticas complexas em substâncias húmicas. Assim, a concentração de radicais livres do tipo semiquinona ( ${ }^{\circ}$ spins), que pode ser mensurada por meio da espectroscopia de ressonância paramagnética eletrônica (EPR), é uma propriedade da matéria orgânica do solo que está relacionada com o seu grau de humificação (Senesi, 1990a,b).

Há forte correlação positiva entre a concentração de spins e a aromaticidade (Senesi, 1990a,b; MartinNeto et al., 1994, 1998). Então, a concentração de spins da matéria orgânica do solo deve diminuir com o aumento da declividade e com a conseqüente diminuição da estabilidade em diferentes posições de uma vertente.

No presente trabalho, foram testadas as seguintes hipóteses: o acúmulo e o grau de humificação da matéria orgânica do solo aumentariam com a diminuição da declividade em vertentes latossólicas; e a quantidade de carvão no solo e sua idade também aumentariam com a diminuição da declividade. A primeira hipótese foi testada correlacionando a declividade com o teor de $\mathrm{C}$ dos solos e com o grau de humificação dos ácidos húmicos. A segunda hipótese foi testada correlacionando a quantidade de carvão do solo e suas idades radiocarbônicas com a declividade. Considerando que a declividade é um atributo do relevo que está relacionado com a acumulação e a estabilidade da matéria orgânica no solo, o objetivo deste trabalho foi avaliar quantitativamente relações entre a declividade, o grau de humificação dos ácidos húmicos e o conteúdo e a idade radiocarbônica dos fragmentos de carvão.

\section{MATERIAL E MÉTODOS}

\section{Caracterização da área estudada}

Neste trabalho foram utilizados quatro solos sob pastagens de uma área cratônica do Sul de Minas Gerais, com latitude sul entre $21^{\circ} 37^{\prime} \mathrm{e} 21^{\circ} 41^{\prime} \mathrm{e}$ longitude oeste de Greenwich entre $44^{\circ} 51$ 'e $44^{\circ} 57$ ' (Figura 1). O clima é subtropical moderado úmido, de acordo com a classificação de Köppen. Os solos apresentam regime hídrico údico e regime térmico isotérmico (Resende et al., 1988) e são originados de gnaisses do Complexo Cristalino, do Pré-Cambriano.

Esses solos correspondem a uma toposseqüência comumente encontrada na Superfície Dissecada de Topografia Montanhosa (Camargo et al., 1962), cujo afeiçoamento teria se iniciado no Plioceno, com o recuo das encostas e a instalação da rede de drenagem. Os mecanismos que ativaram o intemperismo a a erosão/ dissecação teriam sido as alternâncias entre climas

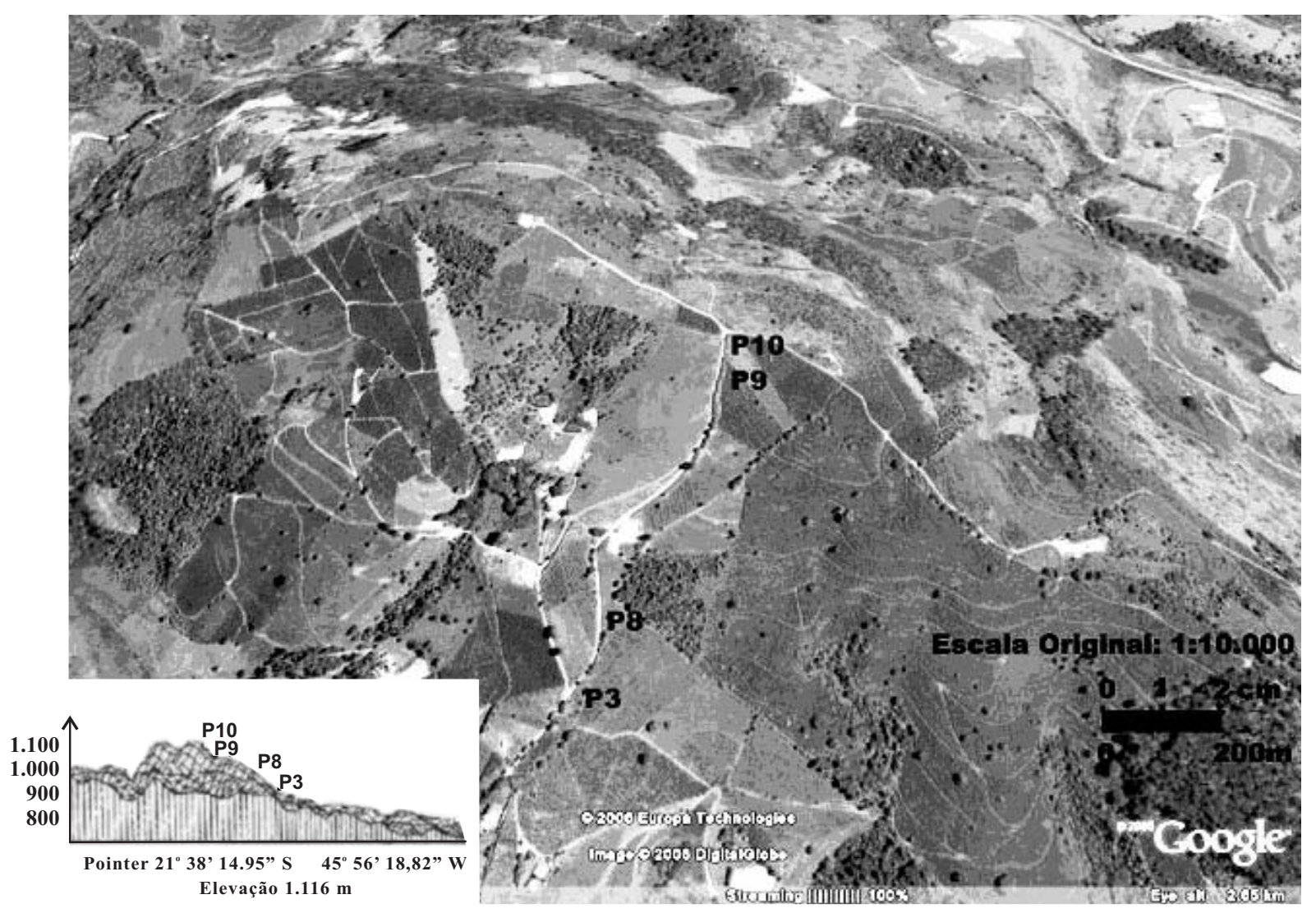

Figura 1. Localização da área do estudo. 
úmidos e semi-áridos (Bigarella et al., 1965a,b) e os movimentos tectônicos residuais, persistentes até os dias atuais (Mioto, 1984, 1990; Saadi, 1991). Os movimentos tectônicos seriam reponsáveis por movimentos de massa que transportaram a cobertura latossólica a curta distância. Silva \& Vidal Torrado (1999) e Silva (1997) reportaram a presença de espelhos de falha no saprolito e no horizonte $\mathrm{Bw}$, bem como mudanças na mineralogia da fração argila e na relação $\mathrm{Ti} / \mathrm{Zr}$ em profundidade em P3, que constituem evidências da ação da tectônica ressurgente na região estudada.

Os perfis dos solos estudados (incluindo horizontes e sub-horizontes) foram descritos, amostrados e classificados nas posições de topo (P10), ombro (P9), meia encosta (P8) e meia encosta/sopé (P3) (Figura 1), de acordo com Embrapa (1999a): Latossolo Vermelho distrófico húmico textura muito argilosa (P10), Latossolo Vermelho distrófico típico textura muito argilosa (P9), Latossolo Vermelho-Amarelo distrófico típico textura muito argilosa (P8) e Latossolo Vermelho-Amarelo distrófico húmico textura muito argilosa (P3). Esses solos apresentam a seguinte classificação, de acordo com a Soil Taxonomy (Soil Survey Staff, 1994): Humic Rhodic Hapludox, Rhodic Hapludox, Xantic Hapludox e Humic Xantic Hapludox. No quadro 1 são apresentados atributos da paisagem e dos solos estudados.

Quadro 1. Horizontes, profundidades, posição na encosta, declividade, teor de argila, pH, soma de bases (SB), capacidade de troca de cátions (CTC), carbono, nitrogênio e relação C/N de amostras de solo de horizontes e sub-horizontes de Latossolos

\begin{tabular}{|c|c|c|c|c|c|c|c|c|c|c|}
\hline Horizonte & Profundidade & $\begin{array}{c}\text { Posição } \\
\text { na encosta }\end{array}$ & Declividade & Argila & $\begin{array}{c}\mathbf{p H} \\
\mathrm{H}_{2} \mathrm{O}\end{array}$ & SB & CTC & $\mathbf{C}$ & $\mathbf{N}$ & $\begin{array}{c}\text { Relação } \\
\mathrm{C} / \mathrm{N}\end{array}$ \\
\hline & $\mathrm{cm}$ & & & \multicolumn{2}{|l|}{$\mathrm{g} \mathrm{kg}^{-1}$} & \multicolumn{2}{|c|}{$-\mathrm{cmol}_{\mathrm{c} \mathrm{kg}} \mathrm{kg}^{-1}$} & \multicolumn{2}{|c|}{$-\mathrm{g} \mathrm{kg}^{-1}$} & \\
\hline \multicolumn{11}{|c|}{ Latossolo Vermelho distrófico húmico (Humic Rhodic Hapludox) } \\
\hline $\mathrm{Ap}$ & $0-32$ & & & 475 & 5,6 & 3,89 & 7,77 & 37,7 & 2,2 & 16,9 \\
\hline A2 & $32-65$ & $\mathrm{P} 10$ & $1,15^{\circ}$ & 565 & 5,6 & 1,50 & 11,73 & 24,4 & 1,2 & 20,2 \\
\hline A3 & $65-117$ & Topo & $2 \%$ & 622 & 5,6 & 1,38 & 7,56 & 17,1 & 0,6 & 27,0 \\
\hline $\mathrm{AB}$ & $117-152$ & & & 675 & 5,4 & 1,40 & 6,35 & 16,2 & 0,5 & 29,7 \\
\hline BA & $152-185$ & & & 654 & 5,5 & 1,36 & 5,20 & 14,1 & 0,6 & 24,5 \\
\hline $\mathrm{Bw}$ & $185-300^{+}$ & & & 655 & 5,4 & 1,16 & 4,23 & 12,7 & 0,5 & 23,5 \\
\hline \multicolumn{11}{|c|}{ Latossolo Vermelho distrófico típico (Rhodic Hapludox) } \\
\hline Ap & $0-34$ & & & 560 & 5,6 & 1,78 & 6,02 & 40,0 & 2,6 & 15,4 \\
\hline A2 & $34-65$ & P9 & $2,29^{\circ}$ & 662 & 5,8 & 2,05 & 5,86 & 20,7 & 1,1 & 18,8 \\
\hline $\mathrm{AB}$ & $65-100$ & Ombro & $4 \%$ & 693 & 5,4 & 2,08 & 6,83 & 16,3 & 0,8 & 19,6 \\
\hline $\mathrm{BA}$ & $100-142$ & & & 675 & 5,5 & 1,28 & 4,41 & 12,3 & 0,7 & 17,6 \\
\hline $\mathrm{Bw}$ & $142-190^{+}$ & & & 673 & 5,3 & 1,69 & 4,57 & 09,6 & 0,6 & 16,9 \\
\hline \multicolumn{11}{|c|}{ Latossolo Vermelho-Amarelo distrófico típico (Xantic Hapludox) } \\
\hline Ap & $0-20$ & & & 550 & 4,1 & 2,70 & 9,70 & 33,1 & 2,3 & 14,4 \\
\hline $\mathrm{AB} 1$ & $20-35$ & P8 & $3,43^{\circ}$ & 607 & 4,5 & 2,47 & 10,37 & 23,0 & 1,1 & 21,3 \\
\hline $\mathrm{AB} 2$ & $35-60$ & Meia & $6 \%$ & 650 & 4,7 & 2,18 & 8,47 & 10,0 & 0,6 & 16,2 \\
\hline $\mathrm{BA}$ & $60-105$ & encosta & & 665 & 4,9 & 1,77 & 6,77 & 08,9 & 0,8 & 11,5 \\
\hline $\mathrm{Bw}$ & $105-165^{+}$ & & & 700 & 5,6 & 1,72 & 6,22 & 05,8 & 0,7 & 8,9 \\
\hline \multicolumn{11}{|c|}{ Latossolo Vermelho-Amarelo distrófico húmico (Humic Xantic Hapludox) } \\
\hline Ap & $0-20$ & & & 565 & 5,7 & 8,22 & 12,22 & 24,0 & 1,4 & 17,1 \\
\hline A2 & $20-68$ & P3 & $5,71^{\circ}$ & 625 & 5,0 & 1,61 & 10,41 & 20,8 & 1,0 & 20,8 \\
\hline A3 & $68-113$ & Meia & $10 \%$ & 648 & 4,9 & 2,65 & 15,58 & 14,6 & 0,8 & 18,2 \\
\hline $\mathrm{AB}$ & $113-137$ & encostal & & 648 & 4,7 & 1,36 & 9,11 & 09,0 & 0,6 & 15,8 \\
\hline $\mathrm{BA}$ & $137-160$ & sopé & & 640 & 4,8 & 2,60 & 8,85 & 06,0 & 0,4 & 11,1 \\
\hline Bw & $160-220^{+}$ & & & 674 & 4,6 & 2,20 & 7,20 & 04,9 & 0,5 & 9,4 \\
\hline
\end{tabular}




\section{Amostragem}

Amostras de solo foram coletadas nos diferentes horizontes e sub-horizontes dos quatro perfis, para extração dos ácidos húmicos e determinação da concentração de radicais livres do tipo semiquinona. Os fragmentos de carvão também foram coletados nos diferentes horizontes e sub-horizontes do solo, com auxílio de pinças, em amostras de $10 \mathrm{~kg}$ de solo. Posteriormente, foram lavados, secos em estufa a $65^{\circ} \mathrm{C}$ e pesados. Uma parte foi moída, para determinação dos conteúdos elementares de $\mathrm{C}$ e $\mathrm{N}$, de acordo com Nelson \& Sommers (1996), e a outra foi mantida intacta, para determinação das idades radiocarbônicas.

\section{Determinações analíticas}

As análises granulométricas e químicas de rotina ( $\mathrm{pH}, \mathrm{C}, \mathrm{P}$ disponível, elementos do complexo sortivo e acidez total) foram feitas de acordo com o método descrito por Embrapa (1999b), nos laboratórios de solos da Universidade de Alfenas.

Os ácidos húmicos (HAs) foram extraídos com $0,1 \mathrm{~mol} \mathrm{~L}^{-1}$ de $\mathrm{NaOH}$ em atmosfera inerte de $\mathrm{N}_{2} \mathrm{e}$ precipitado com $6 \mathrm{~mol} \mathrm{~L}^{-1}$ de HCL. Posteriormente, os HAs foram tratados com solução 1:1 de $\mathrm{HCl} / \mathrm{HF}$, para abaixar o conteúdo de cinza a menos de $5 \%$, e liofilizados (Swifit, 1996).

Medidas de EPR para determinação da concentração de radicais livres do tipo semiquinona (RLS) foram realizadas à temperatura ambiente em um espectrômetro EPR Century Varian E-109. Tubos de quartzo do EPR foram preenchidos até $0,5 \mathrm{~cm}$ de altura com amostras de ácidos húmicos. O espectrômetro foi operado em X-Band $(9 \mathrm{GHz})$, utilizando-se as seguintes condições experimentais: campo magnético centrado a $\left(\mathrm{H}_{\mathrm{o}}\right)=3.400$ Gauss, freqüência de modulação $(\mathrm{n})=9.519 \mathrm{GHz}$, janela espectral $(\mathrm{DH})=100$ Gauss, amplitude de modulação $(\mathrm{MA})=0,5$ Gauss pico a pico e força de microondas $(\mathrm{P})=0,2 \mathrm{~mW}$. A concentração absoluta dos sinais de radicais livres, usada para estimar o grau de humificação dos HAs, foi medida utilizando um cristal de rubi como padrão secundário calibrado por meio do pico intenso do Varian, e a área relativa do sinal do EPR foi calculada pela integração da segunda derivada do sinal de absorção.

A composição elementar $(\mathrm{C}$ e N) foi determinada em todas as amostras de solos (fração $<2 \mathrm{~mm}$ de diâmetro), ácidos húmicos e fragmentos de carvão, utilizando um analisador elementar LECO CHN 1000 do Departamento de Edafologia da Universidade de Santiago de Compostela, Espanha.

As datações radiocarbônicas por ${ }^{14} \mathrm{C}$ foram feitas no Center for Isotope Research, Groningen, Netherlands, em fragmentos de carvão de todos os horizontes e sub-horizontes dos Latossolos húmicos e em fragmentos de carvão do horizonte $\mathrm{Bw}$ dos Latossolos não-húmicos.

\section{Determinação da declividade}

A declividade foi determinada em todas as posições da vertente por meio de várias medições, empregandose um clinômetro CST. Os valores obtidos apresentaram amplitude de variação muito pequena.

\section{Análises estatísticas}

Os valores modais da declividade nas diferentes posições da vertente foram correlacionados com os teores máximos, médios e mínimos de $\mathrm{C}$ total de cada perfil de solo e com as concentrações máximas, médias e mínimas de radicais livres do tipo semiquinona dos ácidos húmicos de cada perfil de solo, uma vez que os teores de $\mathrm{C}$ diminuem com a profundidade e as concentrações de RLS variam ao longo do perfil. A ANOVA foi efetuada utilizando-se o software SAEG 6.0, e o valor de $\mathrm{F}$ foi corrigido mediante o programa Fcalc versão 1.1. As regressões foram ajustadas utilizandose o programa TableCurve 3.01 (Jandel Corporation).

\section{RESULTADOS E DISCUSSÃO}

Todos os solos estudados apresentam teores de $\mathrm{C} \mathrm{e}$ $\mathrm{N}$ mais elevados na superfície, diminuindo em profundidade (Quadro 1). Com exceção do horizonte $\mathrm{Ap}$, a relação $\mathrm{C} / \mathrm{N}$ dos solos situados nas posições da vertente de maior declividade (P3 e P8) também diminui em profundidade, mas nos solos das posições de menor declividade (P10 e P9) essa relação aumenta até a base do horizonte $\mathrm{AB}$, diminuindo a partir do horizonte BA, até o Bw. Esse comportamento sugere maior resiliência da matéria orgânica em profundidade, nas posições mais estáveis da paisagem.

Os Latossolos húmicos (P10 e P3) possuem maiores quantidades de carvão em todos os horizontes e subhorizontes e os valores estão em consonância com aqueles obtidos por Pessenda et al. (1996) em Latossolos situados nas proximidades do local desses estudos (Quadro 2). O perfil P10 apresenta as maiores quantidades de carvão, possivelmente por se situar no topo plano, onde as perdas de solo por erosão foram e são comparativamente menores do que em outras posições da vertente. Os Latossolos não-húmicos (P8 e P9) apresentam os menores teores de carvão abaixo de $34 \mathrm{~cm}$ de profundidade.

A variação do teor de C orgânico nos fragmentos de carvão em todos os horizontes dos perfis estudados é pequena (entre 386 e $527 \mathrm{~g} \mathrm{~kg}^{-1}$ de solo-Quadro 2), evidenciando homogeneidade de cobertura vegetal (Behling, 1995) no decorrer do tempo.

Nos Latossolos com horizonte A húmico, os teores de $\mathrm{N}$ dos fragmentos de carvão são mais baixos, diminuindo com a profundidade, enquanto os valores da relação $\mathrm{C} / \mathrm{N}$ do carvão são mais elevados em comparação com os dos Latossolos desprovidos de horizonte A húmico, aumentando com a profundidade. 
Quadro 2. Quantidade, idade radiocarbônica, teores de carbono e de nitrogênio orgânico e relação C/N de fragmentos de carvão de perfis de Latossolos

\begin{tabular}{|c|c|c|c|c|c|c|c|}
\hline \multirow{2}{*}{ Horizonte } & \multirow{2}{*}{ Profundidade } & \multirow{2}{*}{$\begin{array}{c}\text { Posição na } \\
\text { encosta }\end{array}$} & \multicolumn{5}{|c|}{ Carvão do solo } \\
\hline & & & Quantidade & Datação ${ }^{14} \mathbf{C}$ & $\mathbf{C O}^{(1)}$ & $\mathrm{NO}^{(2)}$ & Relação $\mathrm{C} / \mathrm{N}$ \\
\hline \multicolumn{3}{|c|}{$\mathrm{cm}$} & $10 \mathrm{~g} \mathrm{~kg}^{-1}$ & Anos AP & $\underline{ }$ & 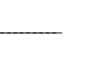 & \\
\hline \multicolumn{8}{|c|}{ Latossolo Vermelho distrófico húmico (Humic Rhodic Hapludox) } \\
\hline Ap & $0-32$ & & 103,4 & $280 \pm 70$ & 450 & 4,8 & 93,8 \\
\hline A2 & $32-65$ & $\mathrm{P} 10$ & 13,9 & $2.100 \pm 80$ & 547 & 4,2 & 130,2 \\
\hline A3 & $65-117$ & Торо & 44,3 & $3.330 \pm 70$ & 496 & 3,5 & 141,7 \\
\hline $\mathrm{AB}$ & $117-152$ & & 8,7 & $4.150 \pm 30$ & 460 & 2,5 & 184,0 \\
\hline $\mathrm{BA}$ & $152-185$ & & 3,7 & $7.200 \pm 50$ & 500 & 2,3 & 217,4 \\
\hline $\mathrm{Bw}$ & $185-300^{+}$ & & 2,7 & & 469 & 2,3 & 203,9 \\
\hline \multicolumn{8}{|c|}{ Latossolo Vermelho distrófico típico (Rhodic Hapludox) } \\
\hline Ap & $0-34$ & & 36,6 & & 414 & 9,0 & 46,0 \\
\hline A2 & $34-65$ & P9 & 3,0 & & 388 & 9,7 & 40,0 \\
\hline $\mathrm{AB}$ & $65-100$ & Ombro & 3,7 & & 437 & 7,5 & 58,3 \\
\hline $\mathrm{BA}$ & $100-142$ & & 2,6 & $3.420 \pm 50$ & 473 & 3,6 & 131,4 \\
\hline $\mathrm{Bw}$ & $142-190^{+}$ & & 1,3 & & 467 & 3,2 & 145,9 \\
\hline \multicolumn{8}{|c|}{ Latossolo Vermelho-Amarelo distrófico típico (Xantic Hapludox) } \\
\hline Ap & $0-20$ & & 14,4 & & 483 & 8,5 & 56,8 \\
\hline $\mathrm{AB} 1$ & $20-35$ & P8 & 3,3 & & 527 & 7,6 & 69,3 \\
\hline $\mathrm{AB} 2$ & $35-60$ & Meia & 3,1 & & 457 & 3,6 & 126,9 \\
\hline $\mathrm{BA}$ & $60-105$ & encosta & 2,2 & & 428 & 3,3 & 129,7 \\
\hline $\mathrm{Bw}$ & $105-165^{+}$ & & 1,4 & $2.210 \pm 50$ & 438 & 2,6 & 168,4 \\
\hline \multicolumn{8}{|c|}{ Latossolo Vermelho-Amarelo distrófico húmico (Humic Xantic Hapludox) } \\
\hline Ap & $0-20$ & & 32,3 & $2.260 \pm 90$ & 433 & 4,7 & 92,1 \\
\hline A2 & $20-68$ & P3 & 17,8 & $3.600 \pm 40$ & 479 & 3,4 & 140,9 \\
\hline A3 & $68-113$ & Meia & 10,8 & $5.330 \pm 50$ & 386 & 2,7 & 143,0 \\
\hline $\mathrm{AB}$ & $113-137$ & encosta/ & 9,1 & $6.850 \pm 50$ & 404 & 2,0 & 202,0 \\
\hline $\mathrm{BA}$ & $137-160$ & sopé & 4,0 & $40.380 \pm 1.035$ & 411 & 1,7 & 241,8 \\
\hline $\mathrm{Bw}$ & $160-220^{+}$ & & 1,9 & $>45.000$ & 431 & 1,7 & 253,5 \\
\hline
\end{tabular}

(1) Carbono orgânico. ${ }^{(2)}$ Nitrogênio orgânico.

A diminuição do teor de $\mathrm{N}$ provavelmente está associada ao maior tempo de interação do carvão com a biota do solo, durante o qual também podem ter ocorrido reações químicas com a solução do solo.

As datações radiocarbônicas dos fragmentos de carvão (Quadro 2) mostram que as idades dos Latossolos com horizonte A húmico são mais elevadas e aumentam com a profundidade, o que evidencia seu maior tempo de interação com a biota do solo. As idades dos fragmentos de carvão do horizonte subsuperficial dos Latossolos não-húmicos são inferiores àquelas encontradas para os mesmos horizontes e profundidades dos Latossolos húmicos.
Enquanto em P10 a idade radiocarbônica dos fragmentos de carvão eleva-se gradualmente com a profundidade, em P3 ela aumenta bruscamente de 2.260 anos AP no horizonte superficial (horizonte Ap) para mais de 40.000 anos AP nos horizontes BA e Bw. O perfil P10 situa-se no topo ( $2 \%$ de declividade) e o perfil P3 situa-se na meia encosta/sopé (10\% de declividade), esta última exposta a processos de erosão e de deposição de materiais provenientes da parte superior da vertente. O aumento brusco da idade radiocarbônica em P3 poderia estar relacionado com movimentos da cobertura pedológica vertente abaixo, provavelmente devido à ação da tectônica ressurgente (Silva \& Vidal Torrado,1999; Silva et al., 2002). 
A figura 2 ilustra os espectros de EPR do radical livre do tipo semiquinona dos ácidos húmicos do subhorizonte A3 de P10 e P3 (Latossolos húmicos), do sub-horizonte $\mathrm{AB}$ de P9 e BA de P8 (Latossolos nãohúmicos), escolhidos por se situarem em profundidades assemelhadas. Os espectros apresentam diferentes intensidades e larguras de linha, refletindo a concentração de radicais livres calculada, que mostra diferenças no grau de humificação da matéria orgânica. Essas diferenças estão associadas aos processos pedogenéticos predominantes (adição e humificação da matéria orgânica em P10 e P9, remoção de solo em P8 e remoção e deposição de solo em P3), aos distintos teores de $\mathrm{C}$ orgânico e aos valores da relação $\mathrm{C} / \mathrm{N}$ desses perfis de solo.

Nos solos situados no topo (P10) e no ombro (P9) da toposseqüência, os teores de radicais livres do tipo semiquinona elevam-se até o final do horizonte A (cerca de $100 \mathrm{~cm}$ de profundidade) e depois diminuem (Quadro 3). No solo da meia encosta (P8), os teores de RLS aumentam com a profundidade, enquanto em P3, situado no segmento da vertente de maior declividade, os conteúdos de RLS apresentam forte oscilação com a profundidade, o que também estaria relacionado, entre outros fatores, com a ação da tectônica ressurgente nessa toposseqüência (Silva \& Vidal Torrado, 1999; Silva et al., 2002).

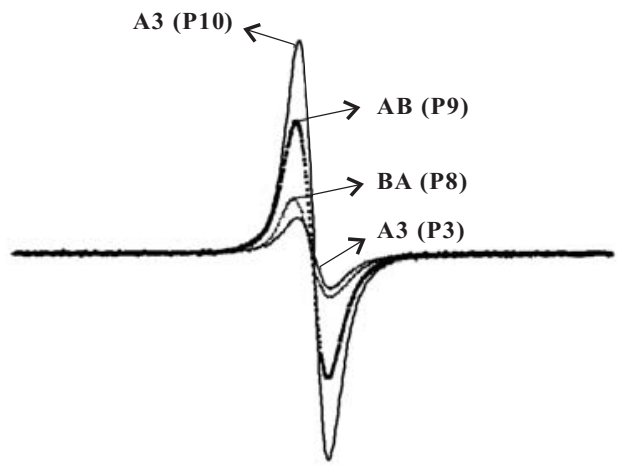

$$
\begin{array}{ccccccccc}
\mathbf{0 , 3 4 2} & \mathbf{0 , 3 4 3} & \mathbf{0 , 3 4 4} & \mathbf{0 , 3 4 5} & \mathbf{0 , 3 4 6} & \mathbf{0 , 3 4 7} & \mathbf{0 , 3 4 8} & \mathbf{0 , 3 4 9} & \mathbf{0 , 3 5 0} \\
\mathrm{H}(\mathrm{T}) & & & & & & & &
\end{array}
$$

Figura 2. Espectros de EPR de radicais livres do tipo semiquinona de ácidos húmicos extraídos de amostras de solo coletadas entre 60 e $100 \mathrm{~cm}$ de profundidade. Condições experimentais: $\mathrm{H}_{\mathrm{o}}: 3458 \mathrm{G} ; \Delta \mathrm{H}: 70 \mathrm{G} ; \mathrm{v}: \mathbf{9 , 4 4} \mathrm{GHz} ; \mathrm{P}: 0,2 \mathrm{~mW}$; T": $25^{\circ} \mathrm{C}$; MA: $1 \mathrm{G}$.

Quadro 3. Conteúdo de radicais livres do tipo semiquinona (média e desvio-padrão), fator g, carbono orgânico,

\begin{tabular}{|c|c|c|c|c|c|c|c|}
\hline \multirow{2}{*}{ Horizonte } & \multirow{2}{*}{ Profundidade } & \multirow{2}{*}{$\begin{array}{c}\text { Posição na } \\
\text { encosta }\end{array}$} & \multicolumn{5}{|c|}{ Ácido húmico } \\
\hline & & & Semiquinona & Fator $\mathrm{g}$ & $\mathrm{CO}$ & NO & Relação $\mathrm{C} / \mathrm{N}$ \\
\hline & $\mathrm{cm}$ & & \multicolumn{2}{|l|}{$\mathrm{n}^{\mathrm{o}} \operatorname{spin} \times 10^{18} \mathrm{~g}^{-1}$} & $-\mathrm{g}$ & - & \\
\hline \multicolumn{8}{|c|}{ Latossolo Vermelho distrófico húmico (Humic Rhodic Hapludox) } \\
\hline Ap & $0-32$ & & $4,31 \pm 0,07$ & 2,0029 & 417,3 & 26,7 & 15,63 \\
\hline $\mathrm{A} 2$ & $32-65$ & $\mathrm{P} 10$ & $6,15 \pm 0,10$ & 2,0029 & 457,4 & 14,5 & 31,54 \\
\hline A3 & $65-117$ & Topo & $7,42 \pm 0,01$ & 2,0029 & 478,1 & 20,5 & 23,32 \\
\hline $\mathrm{AB}$ & $117-152$ & & $6,11 \pm 0,20$ & 2,0008 & 471,7 & 14,2 & 33,21 \\
\hline $\mathrm{BA}$ & $152-185$ & & $5,41 \pm 0,09$ & 2,0030 & 481,2 & 09,7 & 49,60 \\
\hline \multicolumn{8}{|c|}{ Latossolo Vermelho distrófico típico (Rhodic Hapludox) } \\
\hline Ap & $0-34$ & & $3,69 \pm 0,07$ & 2,0030 & 441,8 & 43,3 & 10,2 \\
\hline $\mathrm{A} 2$ & $34-65$ & P9 & $5,24 \pm 0,42$ & 2,0029 & 418,2 & 33,0 & 12,67 \\
\hline $\mathrm{AB}$ & $65-100$ & Ombro & $6,17 \pm 0,11$ & 2,0030 & 480,7 & 21,8 & 22,05 \\
\hline $\mathrm{BA}$ & $100-142$ & & $4,63 \pm 0,04$ & 2,0030 & 502,2 & 13,0 & 38,63 \\
\hline $\mathrm{Bw}$ & $142-190^{+}$ & & $4,18 \pm 0,19$ & 2,0032 & 467,4 & 12,1 & 38,63 \\
\hline \multicolumn{8}{|c|}{ Latossolo Vermelho-Amarelo distrófico típico (Xantic Hapludox) } \\
\hline Ap & $0-20$ & & $3,33 \pm 0,02$ & 2,0032 & 372,3 & 30,7 & 12,12 \\
\hline $\mathrm{AB} 1$ & $20-35$ & $\mathrm{P} 8$ & $3,05 \pm 0,09$ & 2,0032 & 502,4 & 22,4 & 22,42 \\
\hline $\mathrm{AB} 2$ & $35-60$ & Meia & $4,80 \pm 0,03$ & 2,0033 & 284,6 & 51,6 & 5,51 \\
\hline $\mathrm{BA}$ & $60-105$ & encosta & $4,96 \pm 0,02$ & 2,0032 & 692,9 & 34,1 & 20,31 \\
\hline \multicolumn{8}{|c|}{ Latossolo Vermelho-Amarelo distrófico húmico (Humic Xantic Hapludox) } \\
\hline Ap & $0-20$ & & $0,99 \pm 0,05$ & 2,0031 & 431,9 & 41,3 & 10,46 \\
\hline $\mathrm{A} 2$ & $20-68$ & P3 & $2,53 \pm 0,12$ & 2,0031 & 391,3 & 16,1 & 24,30 \\
\hline A3 & $68-113$ & Meia & $2,25 \pm 0,02$ & 2,0031 & 411,2 & 17,2 & 23,91 \\
\hline $\mathrm{AB}$ & $113-137$ & encosta/ & $1,73 \pm 0,08$ & 2,0032 & 489,8 & 18,3 & 26,76 \\
\hline $\mathrm{BA}$ & $137-160$ & sopé & $3,29 \pm 0,04$ & 2,0033 & & & \\
\hline
\end{tabular}
nitrogênio orgânico e relação $\mathrm{C} / \mathrm{N}$ dos ácidos húmicos de perfis de Latossolos 
A concentração de RLS nos ácidos húmicos da maioria das amostras é muito mais elevada do que aquelas reportadas por Rivero et al. (1998) em Oxisol da Venezuela, por Martin-Neto et al. (2001) em Latossolo do Sudeste do Brasil e por González Pérez et al. (2004) em Latossolo do Estado de São Paulo, possivelmente devido ao maior período de tempo de exposição a processos de humificação, uma vez que os perfis estudados se encontram em uma área cratônica. Os valores são também maiores que a faixa de $0 \mathrm{a}$ $2 \times 10^{18}$ spin g ${ }^{-1}$, publicada por Stevenson (1994) como os usualmente reportados para ácidos húmicos de solos. As altas concentrações de RLS encontradas demonstram o elevado grau de humificação da matéria orgânica dos solos estudados.

Os solos situados nas posições mais estáveis da vertente estudada (topo - P10 e ombro - P9) apresentam os maiores teores médios de RLS em relação aos solos situados nas posições sujeitas a sucessivas remoções e deposições da cobertura pedológica. Como a perturbação antropogênica é cronologicamente recente, considerando as idades radiocarbônicas dos fragmentos de carvão (Quadro 2), a topografia possui papel fundamental no processo contínuo de humificação.

O teor de $\mathrm{C}$ dos ácidos húmicos é aproximadamente constante em P10 e P9. Nesses perfis, o teor de N diminui e a relação $\mathrm{C} / \mathrm{N}$ dos ácidos húmicos aumenta com a profundidade (Quadro 3), evidenciando aumento da humificação. O perfil $\mathrm{P} 8$, situado na meia encosta da vertente e que está sujeito à ação de processos de remoção e deposição de materiais de solo, apresenta grandes oscilações nos teores de $\mathrm{C}, \mathrm{N}$ e na relação $\mathrm{C} / \mathrm{N}$ dos ácidos húmicos em profundidade. Essas constatações também se relacionam com a influência da declividade no processo de humificação.

A figura 3 mostra elevadas correlações lineares negativas entre as concentrações de RLS máximas, médias e mínimas e a declividade nas posições da vertente onde se situam os Latossolos estudados. Correlacionaram-se as concentrações máximas, médias e mínimas de RLS de cada perfil com a declividade, a fim de minimizar a influência da variação da RLS em profundidade nos resultados.

$\mathrm{Na}$ figura 4 são apresentadas as elevadas correlações lineares negativas entre os teores máximos, médios e mínimos de $\mathrm{C}$ do solo e a declividade das posições na encosta onde se situam os Latossolos estudados. Também foram correlacionados os teores máximos, médios e mínimos de $\mathrm{C}$ de cada perfil com a declividade, para minimizar a influência da variação dos teores de $\mathrm{C}$ nos resultados.

Os resultados apresentados nas figuras 3 e 4 demonstram a influência da declividade da vertente no teor de C do solo e na concentração de RLS, que diminuem à medida que a declividade aumenta.

As vertentes ocupadas por solos por muito tempo expostos a processos de erosão (Jenny, 1980; Buol et

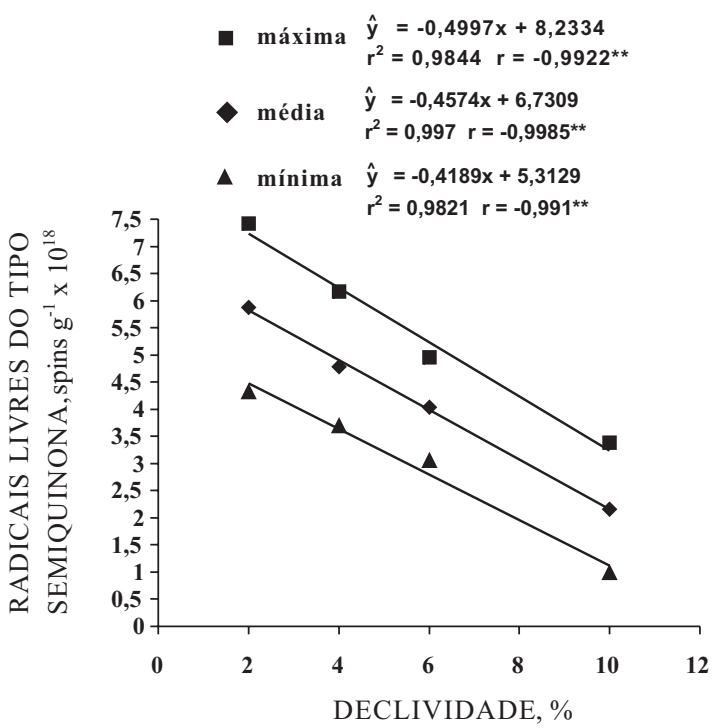

Figura 3. Correlação (r) entre a concentração de radicais livres do tipo semiquinona em amostras de ácidos húmicos e a declividade, equação de regressão e coeficiente de determinação $\left(r^{2}\right)$.

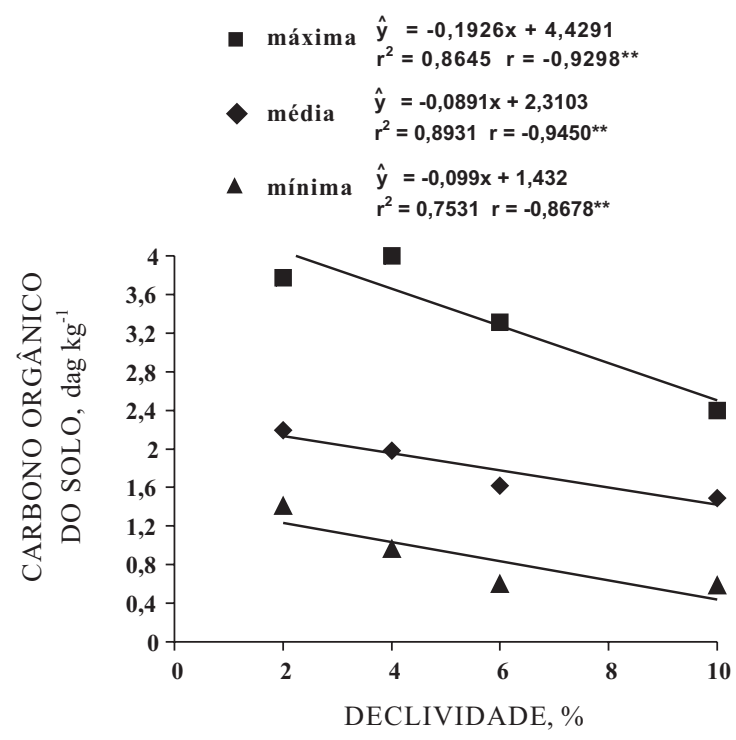

Figura 4. Correlação (r) entre o teor de carbono do solo e a declividade, equação de regressão e coeficiente de determinação $\left(r^{2}\right)$.

al., 1980; Daniels \& Hammer, 1992), que ocorreram de forma mais acentuada em períodos de transição climática (Ledru, 1993, 1996; Behling, 1995; Pessenda et al., 1996; Parizzi et al., 1998; Barberi et al., 2000; Gouveia, 2001; Silva et al., 2004) durante o Pleistoceno tardio e o Holoceno, no Sudeste Brasileiro, possuem maior teor de $\mathrm{C}$ nas posições menos declivosas, que, portanto, foram as mais preservadas da erosão.

O Latossolo húmico (P10) situado no topo da vertente apresenta maiores quantidades de fragmentos 
de carvão em relação ao Latossolo húmico (P3) da meia encosta/sopé, confirmando o modelo de preservação de componentes do solo nas partes mais estáveis da paisagem, proposto por vários autores, como Jenny (1980), Daniels \& Hammer (1992) e Buol et al. (1980). A brusca elevação da idade radiocarbônica (Quadro 2) aliada à elevada oscilação dos valores da concentração de RLS, dos teores de $\mathrm{C}$, de $\mathrm{N}$ e da relação $\mathrm{C} / \mathrm{N}$ dos ácidos húmicos em profundidade (Quadro 3) constituem evidências de que o perfil P3 foi freqüentemente perturbado por processos de deposição e de remoção de materiais do solo.

Os valores das correlações lineares entre as quantidades de carvão, suas idades radiocarbônicas e as declividades não foram estatisticamente significativos, evidenciando independência no comportamento conjunto desses parâmetros.

\section{CONCLUSÕES}

1. Os teores de $\mathrm{C}$ e as concentrações de radicais livres do tipo semiquinona, extraídos de horizontes de perfis situados em diferentes posições da vertente, apresentam elevada correlação negativa com a declividade.

2. A quantidade e a idade dos fragmentos de carvão nos horizontes dos solos estudados independem da declividade e estão relacionadas com suas gêneses e com sua posição nas vertentes.

\section{AGRADECIMENTOS}

À Fundação de Apoio à Pesquisa do Estado de Minas Gerais - FAPEMIG, pelo apoio financeiro para execução de parte deste trabalho. Ao Centro Nacional de Pesquisa e Desenvolvimento da Instrumentação Agropecuária-CNPDIA/Embrapa, pela realização das análises de EPR.

\section{LITERATURA CITADA}

BARBERI, M.; SALGADO-LABOURIAU, M.L. \& SUGUIO, K. Paleovegetation and paleoclimate of "Vereda de Águas Emendadas", central Brazil. J. South Am. Earth Sci., 13:241-254, 2000.

BASSINI, F. \& BECKER, P. Charcoal's occurrence in soil depends on topography in terra firme forest near Manaus, Brazil. Biotropica, 22:420-422, 1990.

BEHLING, H. A high resolution Holocene pollen record from lago do Pires, SE Brazil: Vegetation, climate and fire history. J. Paleolim., 14:253-268, 1995.

BEHLING, H. Late Quaternary vegetation, climate and fire history of the Araucaria forest and campos region from Serra Campos Gerais, Paraná State (South Brazil). Rev. Palaeobot. Palynol., 97:109-121, 1997a.
BEHLING, H. Late Quaternary vegetation, climate and fire history from the tropical mountain region of Morro de Itapeva, SE Brazil. Palaeogeogr., Palaeoclimatol., Palaeoecol., 129:407-422, 1997b.

BERTONI, J. \& LOMBARDI NETO, F. Conservação do solo. 2.ed. Piracicaba, Ceres,1995. 400p.

BIGARELLA, J.J.; MOUSINHO, M.R. \& SILVA, J.X. Considerações a respeito da evolução das vertentes. B. Paranaense Geogr., 16/17:85-116, 1965a.

BIGARELLA, J.J.; MOUSINHO, M.R. \& SILVA, J.X. Pediplanos, pedimentos e seus depósitos correlativos no Brasil. B. Paranaense Geogr., 16/17:117-151, $1965 b$.

BOULET, R.; PESSENDA, L.C.R.; TELLES, E.C.C. \& MELFI, A.J. Une évaluation de la vitesse de l'accumulation superficielle de matière par la faune du sol à partir de la datation des charbons et de l'humine du sol. Exemple des latosols des versants du lac Campestre, Salitre, Minas Gerais, Brésil. Comptes Rendus L'academie Sci., 320:287294, 1995 .

BUOL, S.W.; HOLE, F.D. \& MCCRAKEN, R.J. Soil genesis and classification. 2.ed. Iowa, The Iowa State University Press, 1980. 405p.

CAMARGO, M.N.; HIRANO, C.; FREITAS, F.G.; JACOMINE, P.K.T.; ANTUNES, F.S.; SILVEIRA, C.O. \& BENNEMA, J. Levantamento dos solos da região de influência do reservatório de Furnas. Rio de Janeiro, Ministério da Agricultura, 1962. 462p.

CURI, N. \& FRANZMEIER, D.P. Toposequence of oxisols from the Central plateau of Brazil. Soil Sci. Soc. Am. J., 48:341346, 1984.

DANIELS, R.B. \& HAMMER, R.D. Soil geomorphology. New York, John Wiley \& Sons, 1992. 236p.

EMPRESA BRASILEIRA DE PESQUISA AGROPECUÁRIA EMBRAPA. Centro Nacional de Pesquisa de Solos. Sistema brasileiro de classificação de solos. Brasília, 1999a. $412 \mathrm{p}$.

EMPRESA BRASILEIRA DE PESQUISA AGROPECUÁRIA EMBRAPA. Centro Nacional de Pesquisa de Solos. Manual de análises químicas de solos, plantas e fertilizantes. Campinas, 1999b. 370p.

GONZÁLEZ PÉREZ, M.; MARTIN-NETO, L.; SAABB, S.C.; NOVOTNYA, E.H.; MILORIA, D.M.B.P.; BAGNATOD, V.S.; COLNAGOA, L.A.; MELOE, W.J. \& KNICKER H. Characterization of humic acids from a Brazilian Oxisol under different tillage systems by EPR, 13C NMR, FTIR and fluorescence spectroscopy. Geoderma, 118:181-190, 2004 .

GOUVEIA, S.E.M. Isótopos de carbono na avaliação do remonte biológico de Latossolos e Podzólicos e de eventos paleoclimáticos em distintas localidades do Brasil. Piracicaba, Universidade de São Paulo - Centro Nacional de Energia Nuclear na Agricultura, 2001. 116p. (Tese de Doutorado)

JENNY, H. Factors of soil formation. 3.ed. New York, McGrawHill, 1980. 362p. 
LEDRU, M.-P. Late Quaternary environmental and climatic changes in Central Brazil. Quaternary Res., 39:90-98, 1993.

LEDRU, M.-P.; BRAGA, P.I.S.; SOUBIÉS, F.; FOURNIER, M.; MARTIN, L.; SUGUIO, K. \& TURCQ, B. The last 50,000 years in the Neotropics (Southern Brazil): Evolution of vegetation and climate. Palaeogeogr. Palaeoclimatol., Palaeoecol., 123:239-257, 1996.

LEPSCH, I.F. \& BUOL, S.W. Oxisol Landscape relationships in Brazil. In: INTERNATIONAL SOIL CLASSIFICATION WORKSHOP, 13., Campinas, 1986. Papers. Campinas, SNLCS/Embrapa, USDA, University of Puerto Rico, 1986. p.174-189.

MARTIN-NETO, L.; ANDRIULO, A.E. \& TRAGHETTA, D.G. Effects of cultivation on ESR spectra of organic matter from soil size fractions of a mollisol. Soil Sci., 157:365372,1994 .

MARTIN-NETO, L.; ROSELL, R. \& SPOSITO, G. Correlation of spectroscopic indicators of humification with mean annual rainfull along a temperate grassland climosequence. Geoderma. 81:305-311, 1998.

MARTIN-NETO, L.; TRAGHETTA, D.G.; VAZ, C.M.P.; CRESTANA, S. \& SPOSITO, G. On the interaction mechanisms of atrazine and hydroxyatrazine with humic substances. J. Environ. Quality., 30:520-525, 2001.

MIOTO, J.A. Mapa de risco sísmico do sudeste brasileiro. São Paulo, IPT, 1984. (Monografia, 10 - ITP, 1563)

MIOTO, J.A. Tentativa de zoneamento sísmico do sudeste brasileiro. B. Soc. Bras. Geol. - Núcleo Minas Gerais, 11:3345,1990 .

NELSON, D.W. \& SOMMERS, L.E. Total carbon, organic carbon and organic matter. In: SPARKS, D.L., ed. Methods of soil analysis. Part 3. Chemical methods. Madison, Soil Science Society of America and American Society of Agronomy, 1996. p.961-1009. (Book Series, 5)

PARIZZI, M.G.; SALGADO-LABOURIAU, M.L. \& KHOLER, H.C. Genesis and environmental history of Lagoa Santa, southeastern Brazil. The Holocene, 8:311-321, 1998.

PESSENDA, L.C.R.; ARAVENA, R.; MELFI, A.J.; TELLES, E.C.C.; BOULET, R.; VALENCIA, E.P.E. \& TOMAZELLO, M. The use of carbon isotopes $\left({ }^{13} \mathrm{C},{ }^{14} \mathrm{C}\right)$ in soil to evaluate vegetation changes during the holocene in central Brazil. Radiocarbon, 38:191-201, 1996.

RESENDE, M.; CURI, N. \& SANTANA, D.P. Pedologia e fertilidade do solo: Interações e aplicações. Brasília, MEC/ ESAL/POTAFOS, 1988. 84p.
RIFFALDI, R. \& SCHNITZER, M. Electron spin resonance spectrometry of humic substances. Soil Sci. Soc. Am. Proc., 36:301-305, 1972

RIVERO, C.; SENESI, N.; PAOLINI, J. \& D'ORAZIO, V. Characteristics of humic acids of some Venezuelan soils. Geoderma, 81:227-239, 1998 .

SAADI, A. Ensaio sobre a morfotectônica de Minas Gerais. Belo Horizonte, Universidade Federal de Minas Gerais, 1991. 160p. (Tese - Professor Titular)

SANFORD, R.L.; SALDARRIAGA, J.; CLARK, K.E.; UHL, C. \& HERRERA, R. Amazon rain forest fires. Science, $227: 53-55,1985$.

SENESI, N. Molecular and quantitative aspects of the chemistry of fulvic acids and its interactions with metals ions and organic chemicals. Part I. the electron spin resonance approach. Anal. Chim. Acta, 232:51-75, 1990a.

SENESI, N. Molecular and quantitative aspects of the chemistry of fulvic acids and its interactions with metals ions and organic chemicals. Part II. The fluorescence spectroscopy approach. Anal. Chim. Acta, 232:77-106, $1990 \mathrm{~b}$.

SILVA, A.C. Dinâmica da cobertura pedológica de uma área cratônica do Sul de Minas Gerais. Piracicaba, Escola Superior de Agricultura Luiz de Queiroz, 1997. 191p. (Tese de Doutorado)

SILVA, A.C.; VIDAL TORRADO, P. Gênese dos Latossolos húmicos e sua relação com a evolução da paisagem numa área cratônica do Sul de Minas Gerais. R. Bras. Ci. Solo, 23:329-341, 1999

SILVA, A.C.; VIDAL TORRADO, P.; MACIAS VÁZQUEZ, F. \& SAADI, A. Relações entre solos e paisagens no sul de Minas Gerais. Geociências, 21:97-111, 2002.

SILVA, A.C.; VIDAL TORRADO, P.; MARTINEZ-CORTIZAS, A. \& GRACIA-RODEJA, E. Solos do topo da Serra São José (Minas Gerais) e suas relações com o paleoclima no sudeste do Brasil. R. Bras. Ci. Solo, 28:345-363, 2004.

STEVENSON, F.J. Humus chemistry: Genesis, composition, reactions. New York, John Wiley, 1994. 496p.

SWIFIT, R.S. Organic matter characterization. In: SPARKS, D.L., ed. Methods of soil analysis Part 3. Chemical methods. Madison, Soil Science Society of America and American Society of Agronomy, 1996. p.1011-1069. (Book Series, 5)

SOIL SURVEY STAFF. Keys to soil taxonomy. 6.ed. Washington, USDA Natural Resources Conservation Service, US Govt. Printing Office, 1994. 306p. 